\title{
Concept of a transductive link
}

\author{
Kim Svensson $\odot^{*}$ and Urban Eriksson $\odot$ \\ Lund University Physics Education Research (LUPER) group, Department of Physics, Lund University, \\ SE 22100 Lund, Sweden
}

(Received 17 July 2020; accepted 23 November 2020; published 23 December 2020)

\begin{abstract}
This theoretical paper defines and explores the concepts of transductive links and transductive chains, as part of the theoretical framework of social semiotics. Social semiotics stems from the multimodal framework, which provides a theoretical perspective, constructs, and a language to describe a shift of semiotic material within or between semiotic systems, such as rewriting a text or moving from a function to a plot. Within this framework a shift of semiotic material between two such systems is referred to as a transduction. This paper aims to expand on the concept of transduction by identifying a theoretical contribution to the modeling of this process, referred to as a transductive link. This link is suggested to affect the transduction process and the resulting learning experience. For example, when plotting measurement data, a computer program can be employed to read the data and to transform the data into pixel information. In this case, programming, or the act of programming, acts as a link between the two resources in the transduction process - a transductive link. In other cases, multiple transductions can be performed one after another resulting in these links creating what we define as a transductive chain. By observing and analyzing the use of different semiotic systems in different learning situations, transductive links and chains can be identified and examined. From this identification one has the possibility to find weak links in the transductive chain and address them accordingly. As such, we suggest that transductive links and chains are powerful tools to be able to understand students' learning experiences.
\end{abstract}

DOI: 10.1103/PhysRevPhysEducRes.16.026101

\section{INTRODUCTION}

This is a theoretical paper, building on empirical work, that aims to expand the theoretical framework of social semiotics and multimodality by discussing the concept of a transductive link. This concept provides a descriptive term to be used in the analytical process of learning situations, but also as a way of scaffolding and varying the transduction process in the classroom, which in turn may lead to learning outcomes.

\section{A. Transductions and physics-examples from the discipline}

Physicists constantly use different methods and scientific processes to analyze and investigate different phenomena. In this process, various types of representations are used to discover and enhance different aspects of the phenomena. In this paper we will discuss this using a well-known example from astrophysics: the Hertzsprung-Russell (HR)

\footnotetext{
Kim.Svensson@fysik.lu.se
}

Published by the American Physical Society under the terms of the Creative Commons Attribution 4.0 International license. Further distribution of this work must maintain attribution to the author(s) and the published article's title, journal citation, and DOI. Funded by Bibsam. diagram. An astrophysicist investigating stars and the stellar life cycles is probably going to construct a HR diagram of newly obtained data as part of the analysis process. The process of arriving at this diagram requires a number of precise steps-record data from stars, perform statistical analysis on the data to weed out errors, organize and categorize the data, and finally visualize the data using a scientific visualization tool. Figure 1 showcases some of these steps. Each step requires some expert disciplinary knowledge to perform, such as programming the satellite, constructing the detector, or performing the statistical modeling. Any astrophysicist aiming to fully understand the nature of the stars is required to understand these steps in full. The astrophysicist must understand how the signal from the stars have been manipulated to get a full understanding of what the final representation-the HR diagram - actually represents. These steps are within physics education research (PER) known as transductions [1-5] and describe the process of moving from one type of representation to another-such as moving from the data to the visualization. Similar processes, i.e., transductions, are ingrained in any physicist's work to investigate and understand different phenomena and, similarly, must also be part of the learning process for students. A student must learn how to move between different representations as part of their path towards understanding. Transductions have also 


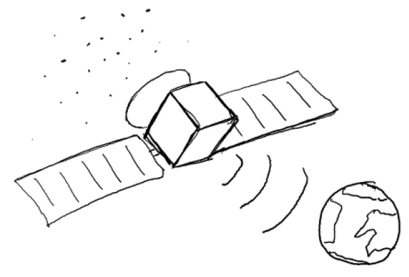

(a)

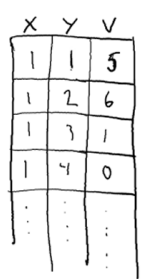

(b)
FIG. 1. (a) Light from the stars is detected by the satellite and sent back to earth as digital signals. (b) The data is converted into an image that is designed to allow the physicist to see and understand certain aspects of the stars.

been shown to play an important role in physics students' meaning-making process; see, for example, Refs. [3,4], and, in particular, Ref. [6] for pertinent examples concerning the unpacking of the HR diagram versus its disciplinary and pedagogical use.

A step that students must understand in order to understand the connection between phenomenon and formula is the step from a laboratory activity to a formula or plot. They must understand how a specific apparatus records the data, how the data are manipulated, and how the data are related to the formula. When students measure the gravitational acceleration using a ticker-tape setup, see Fig. 2, they must understand how the ticker works, why the dots are spaced out, and how to move from the dots to the formulas for velocity and acceleration to determine the gravitational acceleration. This is an example of where a physical concept is transducted using the ticker-tape setup. The laboratory equipment allows for the discernment of a specific aspect of gravity using a certain technique and filters out other aspects. In Fig. 2 the different transductions are measurement $\rightarrow$ calculations $\rightarrow$ formula $\rightarrow$ graph. Each step in the process requires the moving, filtering, and manipulation of semiotic material.

With these examples, we have now highlighted what the normal process of doing and learning science entails.

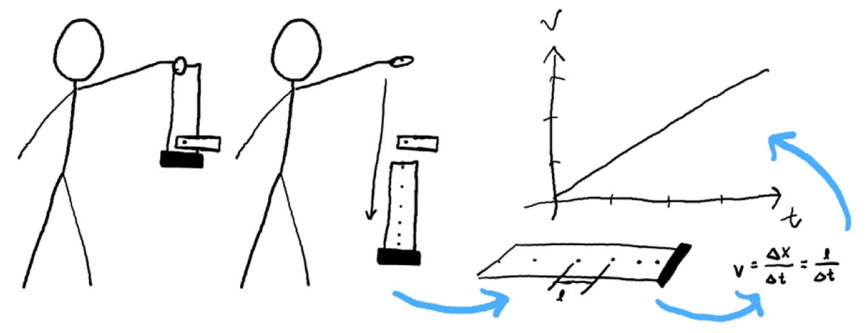

FIG. 2. A tape with a weight is dropped through a ticker. The ticker marks the tape at regular time intervals as the tape falls. The student must then connect the distance between the dots on the tape to the notion of velocity and plot the velocity. From the slope of the velocity in the plot, the student may then calculate the acceleration. These steps are all examples of different transductions.
However, and as we will see, the process of moving from one way of representing information, using a particular semiotic system, to others, has not been addressed properly previously in the social semiotic framework for physics education. Using the concept of transduction, we will thus provide a concept for how these changes can be theoretically described as links and chains, hence contributing to the theory of social semiotics.

\section{BACKGROUND}

The process of transduction has been identified as an important process for students to master as they move towards fluency in the physics discipline $[3,4,7]$. Several theoretical frameworks address this process and take their own view on it [8-10]. Transduction in the way that we use it in this paper stems from the multimodality framework $[11,12]$, a framework which describes how meaning making takes place using several different modes, where a mode is, for example, speech, text, images, gestures, or any other distinct way of representing a concept. By combining modes, such as using text and images in a book, multimodality aims to describe and provide an enhanced learning situation where students move between different modes. Each mode aims to highlight or present certain aspects of the whole concept and it is the combination of modes that provides the student with the opportunity for simultaneous discernment of the different aspects of the concept. Within each mode a concept is represented using that mode's specific attributes. A physics concept may be described in words, as an image, or even an animation, where each of these is a representation of the concept, but each representation holds different potentials for meaning making, often referred to as disciplinary affordances $[6,13,14]$. For example, using the ticker-tape example discussed earlier, the dotted paper strip captures certain aspects of the concept under study, the table created from measuring the data points holds other aspects. The same is true for the graph created from the table and the final formula created from the slope of the graph. Each one of these representations in itself is not enough for fully understanding the phenomenon, but together they create a whole that enhances the possibilities for meaning making by the student.

Social semiotics is a multimodal theory but adds the social aspect as an important aspect of the learning process. Instead of only talking about representations, social semiotics talks about semiotic resources. A semiotic resource is any resource that is used to communicate meaning, such as activities, tools, and representations [15]. Social semiotics studies the meaning-making of specialized groups in society, such as how physicists communicate and make meaning as they discuss and investigate different physical phenomena. Social semiotics, building on the multimodal theory, has taken on the language of multimodality and is using the term "transduction" to mean a shift from one 


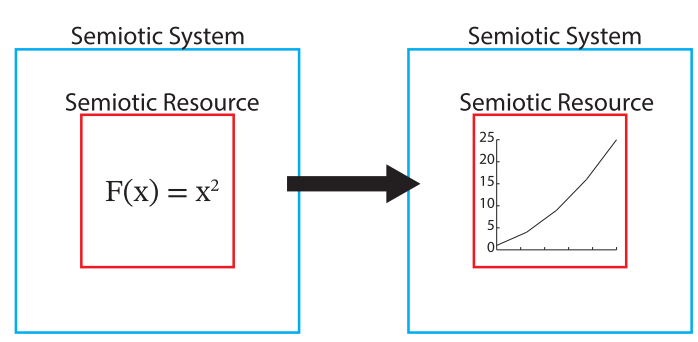

FIG. 3. The transduction process, shifting the semiotic material from a semiotic resource (red) by constructing a new semiotic resource in a new semiotic system (blue). We aim to expand on the description of the arrow (the transduction process) by the theoretical concept of the transductive link.

semiotic resource to another, but also a shift from one semiotic system to another. A semiotic system can be seen as a mode from multimodality, and shifting between different systems is the same as shifting between different modes $[5,16]$. In Fig. 3 we see a very common transduction, namely, the shifting from a formula to a graph. This transduction is described in social semiotics as a shift of semiotic material from a semiotic resource in the semiotic system "formula" into a new semiotic resource in the semiotic system "graph."

However, the process of transduction may be performed between many different semiotic systems and each type of transduction is possibly different compared to any other type. For example, going from a text to a formula is different compared to moving between a formula and a graph. To only describe these different types of transductions using a single word does not capture the breadth of the different types of transduction processes that exists, nor how different transductions may differ. An expansion and understanding of the transduction process is required to be better able to understand the affects and aspects of different transductions.

Transductions are useful tools to showcase a concept in a new way, for example, by drawing a graph that represents a function. This new way of presenting the information contained in the function may change how the students understand the information and the relationship it has to the situation or the discipline. This change in how a person sees and understands a concept is referred to as conceptual change [17-21] and attempts to capture the idea that the understanding of concepts and relationships changes over time as new information and new ways of thinking about it are experienced. By better understanding transductions and its potential role in the conceptual change process, one can get a better understanding of the learning process. Transductions should also be performed by the students as part of their own problem solving and investigation, as they, in the process, necessarily must construct their own representations of the concept. Student created representations have been studied in Refs. [8-10,22] as part of the learning process.

\section{A. Different ways to represent a concept}

In Ref. [7], Airey and Linder describe what they call a "multifaceted way of knowing" a concept. They say that a concept has several ways of being experienced or investigated, such as representing the concept as a formula or as a graph. The different ways of representing the concept provide access to a different facet of the concept itself. They go on to argue that a concept requires a multifaceted way of knowing it before it can be fully understood, i.e., no single representation can convey all the information needed to fully present the concept in question. By representing the concept using different semiotic systems and resources, different facets of the concept can be presented and offer discernment of new aspects of it. However, Airey and Linder also describe a secondary aspect of their constructa link-which aims to connect two different semiotic systems. This link is later referred to as a "transductive link" in [15]. In Fig. 4, the semiotic system "diagrams" acts as the transductive link between "experimental work" and "mathematics." The blue lines in the figure represents a shift between semiotic systems, or a transduction, and the red lines represent a semiotic resource representing the concept. In reality, a concept has many more facets than the six shown by the hexagon in Fig. 4 and there may be many facets that we do not have access to given the semiotic systems we are currently using. However, although the term transductive link is used in Refs. [5,15,16], it is not rigorously defined nor explored.

We claim that how a transduction is performed will have an impact on the meaning-making process; there is a difference between seeing the initial and final semiotic resource compared to understanding the path between them. A transductive link is experienced by the learner and connects the initial and the final semiotic system. We would also like to stress that we are only looking at the actual semiotic resources themselves and what they afford,

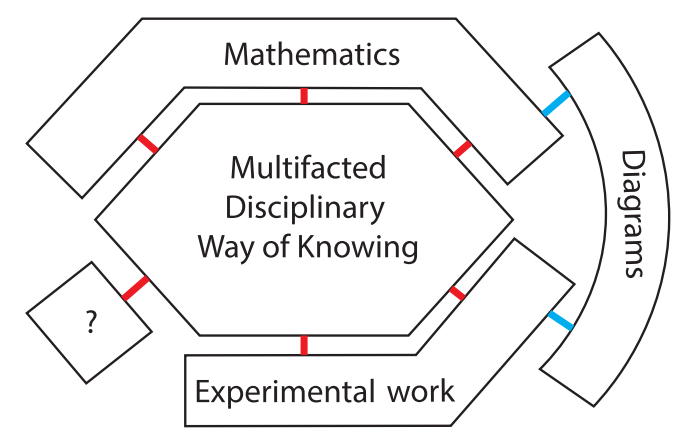

FIG. 4. A concept is experienced using different semiotic systems and many of the concept's facets are revealed through the different types of semiotic systems. Diagrams are used as the transductive link between experimental work and mathematics. The blue lines represents a shift between semiotic systems and the red lines represent a semiotic resource representing the concept. Adapted from image found in Refs. [7,15]. 
as well as how this changes as part of the transduction process. Thus, in this paper, we are not making any claims about students' understanding of a particular transduction process.

The aim of this paper is thus to define and explore the concept of transductive links, introduce transductive chains, and to exemplify how these links and chains can be used in both physics education and physics education research to better teach, understand, and analyze students' meaning-making processes.

\section{TRANSDUCTIVE LINK}

Although introduced in Ref. [15] and later used in Refs. [5,16], no formal definition of a transductive link exists. By building on the description of transduction in Ref. [12], where transduction is described as "the movement of semiotic material from one mode to another," we can construct a definition of a transductive link in a social semiotic setting:

A transductive link is any semiotic system that supports the transduction process between two different semiotic systems.

The word "support" is chosen in this definition because a transductive link and its implementation may come in many different forms and different transductive links will affect the semiotic material differently. Thus, the word support captures the effect and intent of the transductive link. A transductive link should support the transduction and make it, or the semiotic material itself, easier to discern. For example, by using gestures to indicate how a function can be drawn in a graph, we employ the semiotic system of "gestures" to support the transduction process. The gestures will affect the transduction process and help the learner discern new and important aspects of the situation, such as making the connection between a point on the graph and the evaluation of the function but also how to construct and read a $2 \mathrm{D}$ graph.

A semiotic system becomes a transductive link when it is employed with the purpose of supporting the transduction process. Thus, we need an initial semiotic system and a final semiotic system to be able to define a transduction and its transductive link. However, this also allows us to break down the transduction into smaller pieces by stating that the transductive link is our final semiotic system. There is now a transduction from the initial semiotic system to the old transductive link and between them we may find, or use, another transductive link. This reduction will come to an end when no new semiotic system can be found to be a transductive link. Remember that a semiotic system must represent the concept in a qualitatively different way. If we just keep dividing the transduction into smaller steps, we will eventually end up with a change that cannot be described as representing the concept in a qualitatively different way and because of this they are not transductive links.

In another example, Svensson et al. [5] identified programming as a potent transductive link where students created their own simulations of different physical concepts. During the implementation process the students had to unpack and understand the different aspects of the physics involved (semiotic system: formulas) and construct new representations of the physics (semiotic system: interactive simulations) using programming. Figure 5 shows a theoretical example of how a transductive link (programming) can be used to go between two different semiotic systems (here a formula and a graph). In the case of programming, the transduction process is supported in the sense that the use of programming facilitates the entire process and is not a simple addition, such as a gesture, to the process.

As discussed above, the construction of an HR diagram requires a transduction to move from the light emitted from the stars to drawing the diagram. Depending on how the data are processed, and the intent of the usage of the HR diagram, different transductive links may be chosen to be part of the transduction process. In the example from the introduction, with the data and the final visualization, programming is used as the transductive link. However, the HR diagram may be constructed without the actual data by an experienced instructor. The instructor may choose to draw the HR diagram on the whiteboard and qualitatively showcase the structure of the diagram, or they may show it in a textbook. In these cases, different transductive links will be used and the resulting semiotic resource will be different with different qualities. In Fig. 6 two different HR diagrams have been constructed using the same data but with different intent. One diagram is designed to showcase the use of an HR diagram and shows a small subset of representative stars while the other has grouped stars together and shows them as circles. The circle radius is an indication of the variability in the absolute magnitude of the star itself. In both cases, programming was used as the transductive link, but how it was applied differed depending on the intent of the final semiotic resource.

\section{A. Transductive chain}

The definition of a transductive link, which we suggested earlier in the paper, allows for the use of several links in the transduction process. We suggest that when several links are used together to support the transduction, this

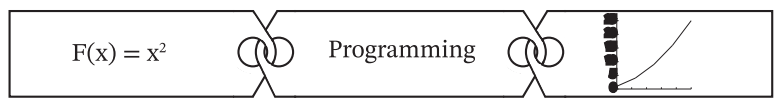

FIG. 5. Programming acts as the transductive link between the mathematical function (semiotic system: formulas) and the semiotic system graph. Here, it is through the use of programming that the transduction takes place. 


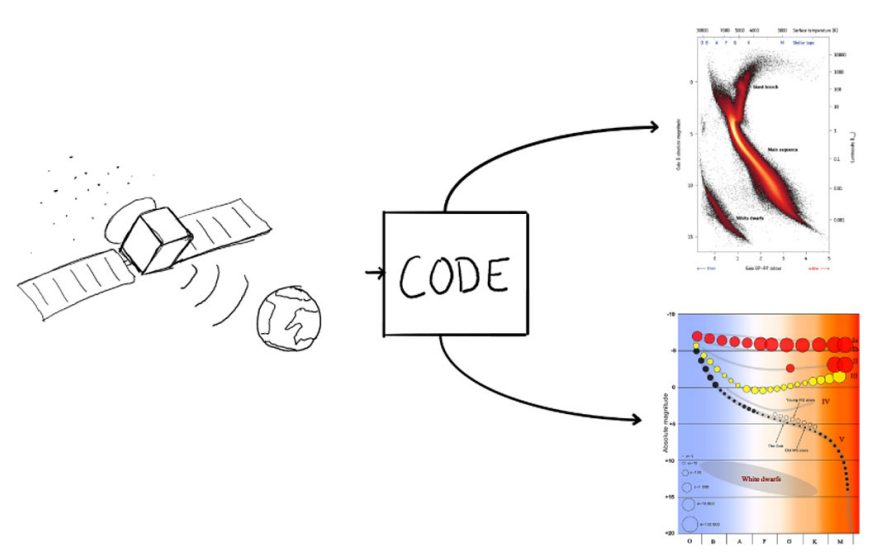

FIG. 6. The same data are captured using satellites and used in a program to construct two different HR diagrams. Depending on how programming is applied will affect the outcome. The top diagram is from the GAIA project [23] and showcases four million stars. The bottom diagram showcases groups of stars and their variation in absolute magnitude is coupled to the size of the circles. The bottom diagram is reprinted, with permission, from Ref. [24]. Both diagrams were constructed using data from satellites and by using programming to visualize it. Using the transductive link, programming, two different semiotic resources were constructed from the same data but they aim to showcase different aspects of the data.

combination forms a transductive chain (Fig. 7). A transductive chain may be composed of just a few links to form a short transductive chain, or it can consist of many different transductive links in a longer chain. A long transductive chain could be a physics project in class, where the project starts with a problem statement and ends in a report or presentation (the in between transductive links are, for example, laboratory equipment, diagrams, mathematics, speech, gestures, text). This whole project can be seen as a transduction from the stated problem to the report through the use of a chain of transductive links.

A transductive chain may be built up over time as new insights are obtained through different transductions. At the end of a transduction process, the initial semiotic material has been shifted to a new semiotic system with the construction of a new semiotic resource in that system. This new semiotic resource may provide new insights or ideas for further study or experimentation, such as if measured data do not line up with theoretical predictions, which then triggers the development of new models and theories. The new semiotic system is used as the transductive link for taking the next step in the exploration process, thus extending or creating a transductive chain.

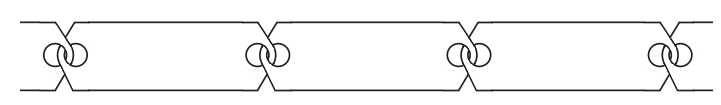

FIG. 7. A transduction can often be divided up into several transductive links, as seen in the figure, forming a transductive chain.
We suggest that this process of expanding the transductive chain by using a previous semiotic system as a stepping stone in the shift towards a new semiotic system (and a new semiotic resource) allows us to theoretically describe the flow of semiotic material in different learning situations.

Once again, to construct the HR diagram, it may be necessary to perform several different steps, e.g., obtaining the data from the satellite, performing different statistical operations, obtaining new values from it through different formulas and visualizing the data in the diagram. This would be an example of a transductive chain, where several links are employed after each other, or at the same time, to produce the diagram. The HR diagram may not be the end of the chain. Instead it may just act as a stepping stone to another semiotic resource which is better suited to understand a new phenomenon that could only been seen in the HR diagram. The HR diagram may only be there to provide some insight and this insight sparks the creation of a new diagram, simulation, formula, or paragraph in a chapter. In this case, the HR diagram acts as a transductive link for this new semiotic resource. It should be noted that any semiotic system is intended to become a transductive link to another one. Any new insight gained from the semiotic resource should trigger further exploration into the new thought and will require the construction of new semiotic resources. The GAIA satellite data was plotted in a HR diagram, and new, or unexpected, structures were found in the distribution of white dwarfs which lead to further research (see, for example, Ref. [25]). The HR diagram became a transductive link in the transduction process for new research after new insight had been discerned in the diagram.

\section{DISCUSSION AND IMPLICATIONS}

Below follows a discussion of the use of transductive links as well as implications based on and around the concept of a transductive link. Suggestions of how transductive links may be used to inform and understand different learning situations are given and examples of transductive links from research literature are highlighted. By giving enlightening examples of how to approach and use transductive links in research, or in teaching, we believe that the concept itself can provide a new way of thinking about, and approaching, different learning situations in physics education.

\section{A. Transductive link as a descriptive term for analysis}

In qualitative physics education research it is often required to analyze different learning scenarios and create rich descriptions of the students' interactions and discussions. This rich description then acts as the basis of the analysis of how to interpret and improve the learning situation. By identifying any transductions or transductive links used by the instructor(s), or the students, the description of the data becomes richer and more detailed, see 
Sec. IV C for examples of transductive links in research literature. One way that the richness of the descriptions can be increased is by the potential of forming categories of transductive links.

\section{Categorization of transductive links}

When transductions or transductive links have been identified, a possible further step in the analysis process is to categorize different transductive links-also their uses-into categories. For example, programming could be used as a transductive link in both a pedagogical and disciplinary way, depending on how it is applied. As described in Refs. [6,14], a single semiotic resource may have different pedagogical and disciplinary affordances depending on how it is used. The same is true for transductive links; depending on how they are applied, they will afford pedagogical or disciplinary aspects; this can be seen in Fig. 6 where the same transductive link is applied with different intent. In two different papers, Svensson and colleagues demonstrated, both practically [16] and theoretically [5], how programming may be used as a transductive link to increase the pedagogical affordance when learning about Newton's laws of motion. They also argue for how programming may be used to increase the pedagogical affordances, both of programming itself, but also of the semiotic resources that are created using programming. To increase, or to use programming with a pedagogical intent, the authors argue that the students, and instructors, should use programming's ability for quick and easy iterations to explore and vary different aspects of the simulation but also programming's ability to construct precise visualizations based on hidden data such as visualizing "temperature" as a color. When these aspects are used to explore and understand different physical concepts, such as the connection between position, velocity, and acceleration, we say that programming is used as a transductive link with pedagogical intent. Whereas in Ref. [25] programming is used with disciplinary intent and aim to highlight different disciplinary aspects of HR diagrams so that any discrepancy between data and theory can be identified.

Further, each transductive link will also have some inherent aspects that affect the transduction process. For example, the programming of a simulation allows the possibility for easy quantitative manipulation of numbers, whereas a drawing on the whiteboard allows for quick and easy exaggeration of different qualitative aspects. The various inherent aspects of a transduction can be seen in Ref. [16], where programming, through an update loop, was used to showcase the relationship between position, velocity and acceleration. The first program in Ref. [16] produced a simulation where a ball appears to fall down with an accelerated motion and this visualization allowed for discernment of what the relationship between position, velocity, and acceleration in the update loop actually means. The code for the simulations can be found in
Ref. [26]. This type of discernment may be much harder if the student was presented with a static image or only formulas (see, for example, Refs. [27-30] for studies using animations as learning tools in science education) or, as Ref. [7] describes it: the animation offers discernment of a new facet of the concept.

Transductive links can thus be categorized both in how they are used, but also with respect to their inherent affect on the transduction process. These categories provide a meaningful description of the situation. Instead of just saying "... the data were transducted into a graph..." we can now say "... the data were transducted into a graph using programming as the transductive link with the intent to showcase X..." The intent of the transduction and the transductive link affect the final graph and how the final graph may be used and both need to be presented to fully understand the affect of the transduction itself.

\section{Disciplinary and pedagogical uses of transductive links}

As Volkwyn et al. [3] argued, a transduction acts as a filter and as a highlighter for different disciplinary relevant aspects, such as extracting the intensity of a signal, while not taking the polarization or angle of the signal into account. The purpose of a transductive link is thus to extract and filter the information in the intended semiotic material in order to highlight some chosen aspects. A similar effect is described by Fredlund et al. [14] as part of the unpacking process of semiotic resources. Unpacking a semiotic resource is the act of stripping the resource down to its disciplinary relevant aspects and highlighting only a few, or only one of them, in a pedagogical manner. Here we can see that the act of transduction is very close to the act of unpacking with the difference being that an unpacking does not require a shift between semiotic systems. However, we can say that within a transduction exists the act of unpacking with the added element of constructing a new semiotic resource in a new semiotic system and that the transductive link must help facilitate the unpacking.

In a teaching and learning situation, a teacher will most likely use transductive links with pedagogical intent and aim to construct a new semiotic resource with higher pedagogical affordance than the originally used resource. On the other hand, a researcher, or a disciplinary expert, may use the same transductive links to construct a semiotic resource with high disciplinary affordances, as seen in Fig. 6. When transductive links are used in these ways, it can be argued that they are used to "unpack" the initial semiotic resource. In Ref. [6], the authors describe this unpacking in an example to provide a higher pedagogical affordance (see below) (see Fig. 8 for a schematic representation of the unpacking process). The unpacking of a semiotic resource will take on different characteristics depending on what transductive links that are used and may thus change the resulting pedagogical affordances of 


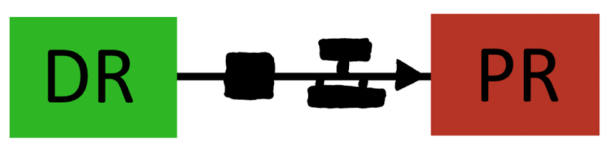

FIG. 8. A semiotic resource with high disciplinary affordances [disciplinary resource (DR)] is unpacked to construct a new semiotic resource with more pedagogical affordances [pedagogical resource $(\mathrm{PR})]$. The unpacking of the semiotic resource may be done using different transductive links, either as complete steps or as scaffolding, if the unpacking requires a transduction.

the new semiotic resource. Airey and Eriksson [6] (p. 1-2) use the following definitions of disciplinary and pedagogical affordances:

Disciplinary affordance: the agreed meaning making functions that a semiotic resource fulfils for a particular disciplinary community.

Pedagogical affordance: the aptness of a semiotic resource for teaching some educational content.

When using a transductive link with pedagogical intent, we aim to construct a semiotic resource with high pedagogical affordance. This means that we must not only understand the physics the semiotic resource aims to showcase, but also understand how to present it in a pedagogical manner. Different transductive links may, and probably should, be used depending on if the outcome is intended for the discipline or for pedagogical purposes.

By using a laser-based measuring device to measure the distance between two objects instead of using an actual measuring tape may reduce the pedagogical aspects of the situation and increase the disciplinary aspects. It may not be important to get an exact measurement, but it may be important to gain a tactile feeling for what it means to measure and how to do it. Thus, a researcher may investigate what a semiotic system provides if it is used as a transductive link, how it may be used, and its potential effect on the semiotic material itself.

\section{B. Scaffolding for instruction}

It is important for teachers and instructors to be aware of the effect that the use of transductions might have in a learning situation. Often instructors need to perform transductions themselves in the classroom, but other times their students need to be able to perform transductions on their own. In such a scenario, one needs to consider the intent of the transduction itself- "What is the purpose of the transduction in this situation?", "What should the transduction filter and what should it highlight?" Once these questions have been answered, the teacher, or the student, needs to choose one, or several, appropriate transductive links that will help facilitate these aspects.

As an example of this, we would like to describe a hypothetical scenario where the instructor has chosen to use speech and gestures as their transductive links.
- Lecturer: Here we evaluate the function at zero [points at the graph at $(0,0)]$ and gets the value 5. [points at the graph at $(0,5)$ and draws a point]

By doing this for all $x$ values we get a line of dots which represents our function. [draws a line in the graph]

The intent of the situation is to showcase how to move between a function and a graph (a transduction from a function to a graph) rather than to use the graph itself to showcase qualitative aspects of the function. To highlight the relationship between the function and the graph, the instructor used gestures and speech as their transductive links. The transductive link is thus used to construct a semiotic resource with high pedagogical affordance. If the intent of the transduction was different, such as having a talk at a scientific conference, there would be no need to use the speech or gestures to support the transduction itself. The graph would probably have been created using a program with the intent to reproduce the function in an accurate manner. The lecturer in the situation above has chosen their transductive links so that they are scaffolding the construction of the new semiotic resource in a pedagogical way for their students. The choice of transductive links to use in an educational setting should, thus, match the intent of the transduction and the ability of the students.

\section{Variation of transductive links}

With the identification of different transductive links comes the possibilities of changing and modifying them and observing the results. An instructor may try out different transductive links, or add new links to their chain, to further filter or enhance different relevant aspects. They may remove a link that they do not think serves its purpose and replace it with another link. This allows the teacher to identify weak links and to vary their teaching. Figure 9 shows a link being replaced with another to change how the semiotic material flows from one semiotic resource to another.

\section{Transductive links and the flow of semiotic material}

Transduction is the reproduction of semiotic material in a new mode. In social semiotics, we say that the transduction constructs a new semiotic resource based on the semiotic material in the initial semiotic resource. Thus, we may describe the transduction process as filtering, enhancing different aspects of the semiotic material, but also as a flow of semiotic material from one semiotic resource to another. The transductive links used in the transduction process are thus used to facilitate this flow of semiotic material, how it changes, how it is modified and how it will be used when a transduction is made. Changing the transductive links will affect the flow of semiotic material and some transductive links may hinder or improve the flow. How the semiotic material changes will affect the resulting semiotic resource because the semiotic resource is just a way of conveying the 


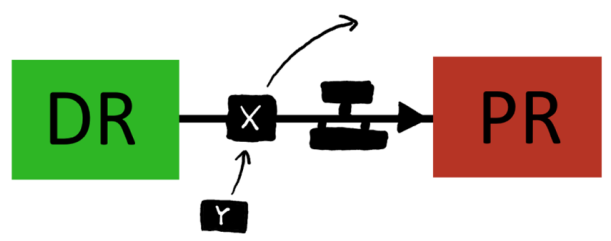

FIG. 9. A transductive link is changed or replaced to construct a new flow of semiotic material from the disciplinary resource (DR) to the pedagogical resource (PR). The new link will affect the transduction process and the affordances of the new semiotic resource.

semiotic material itself. In Fig. 9 we may think of the semiotic material flowing from the disciplinary resource to the pedagogical resource through the transductive link.

\section{Identifying transductive links in literature}

As we have described earlier, the concept of a transductive link has not previously been identified as a critical aspect in the learning process, nor has it been thought of in an analytic way in a PER perspective. By providing an indepth discussion of transductive links, we offer researchers an opportunity to explore this new tool in their own research and to develop it further.

To exemplify how transductive links could be used analytically we have chosen four previously published physics education research articles as examples of how the concept of a transductive link could be employed as part of the analytical and descriptive process. In the first two examples $[5,16]$, programming was identified as a transductive link when trying to learn physics and used as a transductive link between many different semiotic systems. Further, in these two articles, the authors analyse programming itself as a tool for enhancing the meaning making in physics education and identify different aspects of programming that could be useful when employed as a meaning-making tool. Such aspects were the ability of programming to act as a transductive link and the possibility of instant feedback to allow for an iterative approach to the exploratory process. These aspects of programming affect the transduction process when programming is used as a transductive link.

Our third chosen example comes from Ref. [3], who explored and described the role of transduction in science learning, specifically in the physics laboratory, through the use of digital or technical devices. The role of such devices in a physics laboratory (such as a telescope or a voltmeter) is described in terms of how they intensify and filter out different signals. In Fig. 3 in Ref. [3] they show an x-ray signal from outer space being detected by a satellite, and the satellite sending a processed version of the signal down to earth where a graph is produced. This process has filtered out unnecessary information and intensified the specific information that the signal contained, such as its direction, intensity, and wavelength. In this case, we argue that the satellite-earth-system performs the transduction in which mathematics and programming acts as the transductive links. Here, the programming allows the satellite to perform the necessary mathematical operations on the signal to filter out and to intensify the relevant semantic material while the system on earth interprets the signal and further transducts it into, say, a visible graph. Further, we believe that the concept of a transductive link could be used to describe other transductions that they describe throughout the article, but will provide just this single example for the sake of exemplifying the application of transductive links. Volkwyn et al. [3] ends the paper with a discussion about what makes different devices suitable to use for different content and concludes that different types of devices (that allows for transductions in different ways) are better suited for different circumstances. To us, this is an example of how different transductive links affect the possible meaning making in different ways.

Our fourth and final example of how transductive links can be identified from examples in the literature comes from Ref. [31]. In this example, Gregorcic, Planinsic, and Etkina [31] studied students' use of gestures when engaging with an interactive whiteboard through a physics playground program where they were asked to explore and discuss different physical concepts. Gregorcic et al. [31] give an example of a student who is using their hand to show how an object is moving in a circle around another object (the students in this situation are exploring Newton's law of gravitation and are observing different orbits). The student's use of gestures supports their speech as they attempt to move from a verbal description of the situation to a visual image. Thus, this is an example of where gestures are used as a transductive link while supporting the transduction.

Both Refs. [3,31] have rich descriptions of each particular learning situation and identify different aspects of them as having different roles. In these descriptions we find evidence of transductive links, as they are being defined in this paper, and would like to suggest that although the idea of transductive links may not be a new concept per se; it has been "hidden" in the research description. Thus, we propose that transductive links should be acknowledged as a concept for identifying and describing distinct parts of a learning situation, and have, through the above given examples, provided arguments for how transductive links may be used in the analytical process.

\section{CONCLUSIONS}

In this theoretical paper, we have used empirical work to define and explore the concept of a transductive link, as well as its role in a learning situation. We suggest that the concept of a transductive link should be considered an extension of the concept of transduction within social semiotics and multimodality. By providing multiple examples from PER, we show that it is useful for research in physics education. We believe that transductive links play 
an important role in students' learning processes and should therefore be identified as a potent analytic tool to be used when describing and understanding the learning challenges that students encounter in physics.

Different transductive links provide different opportunities for meaning making and the most appropriate transductive link to support the transduction process should be chosen depending on the learning goal of the situation. Further, two or more transductive links can be combined to create a transductive chain. The transductive chain is a natural expansion of transductive links and provides a mental image of how the semiotic material flows through different links before a final semiotic resource is obtained. Each link has its own weaknesses and strengths and affects the semiotic material differently. Transductive links and transductive chains thus allow for a novel description of different aspects of the learning process and the pertinent tools that are used in this process. By identifying the transductive links or chains in a given learning situation, we can begin to study how they affect the transduction process. Weak links may be identified and replaced by better links to improve the teaching and learning experience for the students.

We believe that further analysis of transductive links should aim at identifying how different links affect the transduction process and how the choices of transductive links affects the possibility for learning. This theoretical description of the learning situation will help us to identify and address weak links in students' meaning-making process and may help researchers and teachers to better understand the meaning-making process in physics at large.

\section{ACKNOWLEDGMENTS}

The authors wish to acknowledge the contributions made by Professor Cedric Linder at Uppsala University, whose comments helped shape the structure of the paper. We also want to thank Moa Eriksson at the Lund University Physics Education Research (LUPER) group for her critical reading of the paper.
[1] C. Jewitt, J. Bezemer, and K. O'Halloran, Introducing Multimodality (Routledge, London, 2016), Chap. 4, pp. 59-85.

[2] G. Kress, Multimodality: A Social Semiotic Approach to Contemporary Communication (Taylor \& Francis, London, 2009).

[3] T. S. Volkwyn, J. Airey, B. Gregorcic, and F. Heijkenskjöld, Transduction and science learning: Multimodality in the physics laboratory, Des. Learn. 11, 16 (2019).

[4] T. S. Volkwyn, J. Airey, B. Gregorcic, and F. Heijkenskjöld, in Multimodal transduction in upper-secondary school physics, ISEC 2018, NIE, Singapore (2018), https://www .diva-portal.org/smash/record.jsf?pid=diva2\%3A1081896\& dswid=5683.

[5] K. Svensson, U. Eriksson, and A.-M. Pendrill, Programming and its affordances for physics education: A social semiotic and variation theory approach to learning physics, Phys. Rev. Phys. Educ. Res. 16, 010127 (2020).

[6] J. Airey and U. Eriksson, Unpacking the HertzsprungRussell Diagram: A social semiotic analysis of the disciplinary and pedagogical affordances of a central resource in astronomy, Des. Lear. 11, 99 (2019).

[7] J. Airey and C. Linder, A disciplinary discourse perspective on university science learning: Achieving fluency in a critical constellation of modes, J. Res. Sci. Teach. 46, 27 (2009).

[8] V. Prain and B. Waldrip, A study of teachers' perspectives about using multimodal representations of concepts to enhance science learning, Can. J. Sci. Math. Technol. Educ. 8, 5 (2008).

[9] V. Prain and B. Waldrip, An exploratory study of teachers' and students' use of multi-modal representations of concepts in primary science, Int. J. Sci. Educ. 28, 1843 (2006).
[10] R. Tytler, V. Prain, and S. Peterson, Representational issues in students learning about evaporation, Res. Sci. Educ. 37, 313 (2007).

[11] G. Kress, C. Jewitt, J. Ogborn, and T. Charalampos, Multimodal Teaching and Learning: The Rhetorics of the Science Classroom, Bloomsbury Classics in Linguistics (Bloomsbury Publishing, New York, NY, 2014).

[12] J. Bezemer and G. Kress, Writing in multimodal texts, Written Commun. 25, 166 (2008).

[13] J. Airey, U. Eriksson, T. Fredlund, and C. Linder, On the disciplinary affordances of semiotic resources, in Book of Abstracts: The First Conference of the International Association for Cognitive Semiotics (IACS), September 25-27, 2014 (Lund University, Lund, 2014), pp. 54-55, http://www.diva-portal.org/smash/record.jsf?pid=diva2\% 3A1271202\&dswid $=-8533$.

[14] T. Fredlund, C. Linder, J. Airey, and A. Linder, Unpacking physics representations: Towards an appreciation of disciplinary affordance, Phys. Rev. ST Phys. Educ. Res. 10, 020129 (2014).

[15] J. Airey and C. Linder, Social semiotics in university physics education, in Multiple Representations in Physics Education, edited by D. F. Treagust, R. Duit, and H. E. Fischer (Springer, New York, 2017), pp. 95-122.

[16] K. Svensson, U. Eriksson, A.-M. Pendrill, and L. Ouattara, Programming as a semiotic system to support physics students' construction of meaning: A pilot study, J. Phys. Conf. Ser. 1512, 012026 (2020).

[17] S. Carey, Are children fundamentally different kinds of thinkers and learners than adults?, in Thinking and Learning Skills, edited by S. F. Chipman, J. W. Segal, and R. Glaser (Routledge, New York, NY, 1985), Vol. 2. 
[18] G. J. Posner, K. A. Strike, P. W. Hewson, and W. A. Gertzog, Accommodation of a scientific conception: Toward a theory of conceptual change, Sci. Educ. 66, 211 (1982).

[19] S. Carey, Conceptual Change in Childhood, Bradford Books (MIT Press, Cambridge, MA, 1987).

[20] A. A. diSessa, Metarepresentation: Native competence and targets for instruction, Cognit. Instr. 22, 293 (2004).

[21] S. Carey, Sources of conceptual change, The Jean Piaget Symposium series, in Conceptual Development: Piaget's Legacy, edited by E. K. Scholnick, K. Nelson, S. A. Gelman, and P. H. Miller (Lawrence Erlbaum Associates Publishers, Mahwah, NJ, 1999), pp. 293-326.

[22] V. Prain and R. Tytler, Representing and learning in science, in Constructing Representations to Learn in Science, edited by R. Tytler, V. Prain, P. Hubber, and B. Waldrip (Sense Publishers, Rotterdam, 2013), pp. 1-14, https://doi.org/10.1007/978-94-6209-203-7_1.

[23] ESA/Gaia/DPAC, The GAIA mission (2020), https://sci .esa.int/gaia, Accessed 2020-10-09.

[24] U. Eriksson, Reading the sky: From starspots to spotting stars, Ph.D. thesis, Uppsala University, Physics Didactics, 2014.
[25] S. Cheng, J. D. Cummings, and B. Ménard, A cooling anomaly of high-mass white dwarfs, Astrophys. J. 886, 100 (2019).

[26] K. Svensson, 2D Physics Simulations using Processing IDE for Physics Education Research-Workshop 2018, https://zenodo.org/record/3607528 (2020).

[27] E. S. Paik and G. Schraw, Learning with animation and illusions of understanding., J. Educ. Psychol. 105, 278 (2013).

[28] C. J. Daly, J. M. Bulloch, M. Ma, and D. Aidulis, A comparison of animated versus static images in an instructional multimedia presentation, Adv. Physiol. Educ. 40, 201 (2016).

[29] O. Thomas, Effectiveness of animation and multimedia teaching on students' performance in science subjects, Brit. J. Educ. Soc. Behav. Sci. 4, 201 (2014), https://www .journaljesbs.com/index.php/JESBS/article/view/17100.

[30] A. Koscianski, R. J. Ribeiro, and S. C. R. da Silva, Short animation movies as advance organizers in physics teaching: A preliminary study, Res. Sci. Technol. Educ. 30, 255 (2012).

[31] B. Gregorcic, G. Planinsic, and E. Etkina, Doing science by waving hands: Talk, symbiotic gesture, and interaction with digital content as resources in student inquiry, Phys. Rev. Phys. Educ. Res. 13, 020104 (2017). 